\title{
Comment on "Birt-Hogg-Dubé syndrome in Korean: clinicoradiologic features and long term follow-up"
}

\author{
Hua-Ching Chang ${ }^{1}$, Chih-Wei Sung ${ }^{2}$, and Pai-Chien Chou ${ }^{3,4}$
}

\begin{abstract}
${ }^{1}$ Department of Dermatology, Taipei Medical University Hospital, Taipei; ${ }^{2}$ Department of Emergency Medicine, National Taiwan University Hospital Hsin-Chu Branch, Hsinchu; ${ }^{3}$ Division of Pulmonary Medicine, Department of Internal Medicine, Taipei Medical University Hospital, Taipei; ${ }^{4}$ Division of Thoracic Medicine, Department of Internal Medicine, School of Medicine, College of Medicine, Taipei Medical University, Taipei, Taiwan
\end{abstract}

Received: October 1, 2019 Accepted: December 20, 2019

\section{Correspondence to}

Pai-Chien Chou, M.D.

Division of Pulmonary Medicine, Department of Internal

Medicine, Taipei Medical

University Hospital, No. 252,

Wuxing St, Xinyi District, Taipei

City 110, Taiwan

Tel: +886-2-27372181

Fax: +886-2-23787139

E-mail: baechainchou@gmail.com

https://orcid.org/0000-0002-

9413-9876
We read with interest the research published by Lee et al. [1] in the Korean Journal of Internal Medicine in July 2019 regarding clinicoradiologic features in Korean patients with Birt-Hogg-Dubé (BHD) syndrome. We appreciate the fact that this case series involving 12 Korean patients with BHD syndrome presented characteristics of a higher risk of pneumothorax and less frequent skin lesions compared with previous reports. Skin lesions are the common features and one of the major diagnostic criteria for BHD syndrome, and they usually appear as multiple papules on the face after the age of 20 years. Histologically, the skin tumors are fibrofolliculomas or trichodiscomas [2]. Noticeably, in the Introduction section, Lee et al. [1] cited two articles on Japanese patients with BHD syndrome who presented a lower incidence of skin lesions than Caucasian patients $[3,4]$. However, we observed an unintentional mistake by the authors in the presentation and interpretation of data from the original studies. We adjusted the descriptions of these data in different ways. In a study by Kunogi et al. [3], skin lesions were detected in seven out of 30 patients with BHD syndrome (7/30, $23.3 \%$ ), and fibrofolliculomas were diagnosed in one patient among only two patients undergoing biopsy $(1 / 2$, $50 \%)$. In another study by Furuya et al. [4], 76 individuals among 156 folliculin mutation carriers reported skin lesions (76/156, 48.7\%), but fibrofolliculomas were diagnosed only in six patients from 28 biopsied skin tissues $(6 / 28,21.4 \%)$. These corrected values were still consistent with the relatively lower incidence of skin lesions in Japanese patients. By contrast, one recent study by Iwabuchi et al. [5] in Japan reported a relatively higher incidence of skin lesions in patients with BHD syndrome $(26 / 31,83.9 \%)$ and a high percentage of tissue-proved fibrofolliculomas and/or trichodiscomas (17/23, 73.9\%). The reasons for the high variations in these case series may be attributed to differences in inclusion criteria, possible selection bias, and lack of perception to identify relatively unremarkable skin lesions among Asians. Larger future cohort studies are warranted to obtain more precise data on the prevalence and features of skin lesions in Asian patients with BHD syndrome.

\section{Conflict of interest}

No potential conflict of interest relevant to this article was reported. 


\section{REFERENCES}

1. Lee JH, Jeon MJ, Song JS, et al. Birt-Hogg-Dube syndrome in Korean: clinicoradiologic features and long term follow-up. Korean J Intern Med 2019;34:830-840.

2. Menko FH, van Steensel MA, Giraud S, et al. Birt-HoggDube syndrome: diagnosis and management. Lancet Oncol 2009;10:1199-1206.

3. Kunogi M, Kurihara M, Ikegami TS, et al. Clinical and genetic spectrum of Birt-Hogg-Dube syndrome patients in whom pneumothorax and/or multiple lung cysts are the presenting feature. J Med Genet 2010;47:281-287.

4. Furuya M, Yao M, Tanaka R, et al. Genetic, epidemiologic and clinicopathologic studies of Japanese Asian patients with Birt-Hogg-Dube syndrome. Clin Genet 2016;90:403412.

5. Iwabuchi C, Ebana H, Ishiko A, et al. Skin lesions of BirtHogg-Dube syndrome: clinical and histopathological findings in 31 Japanese patients who presented with pneumothorax and/or multiple lung cysts. J Dermatol Sci 2018;89:77-84. 